IOSR Journal of Pharmacy

ISSN: 2250-3013, www.iosrphr.org

\||| Volume 2 Issue 5 ||| Sep-Oct. 2012\|\| PP.12-18

\title{
Antidiabetic activity of partitionates of Aegle marmelos linn. (rutaceae) leaves ethanolic extracts in normal and alloxan induced diabetic rats
}

\author{
Md. Rafiqul islam khan ${ }^{1 *}$, Md. Ariful islam ${ }^{1}$, Md. Sarowar hossain ${ }^{2}, \mathrm{Md}$. \\ Asaduzzaman $^{2}$, Mir imam ibne wahed ${ }^{1}$, Bytul Mokaddesur rahman ${ }^{1}$, Asm \\ anisuzzaman $^{1}$, Maruf ahmed ${ }^{1}$ \\ ${ }^{I}$ Department of Pharmacy, University of Rajshahi, Rajshahi-6205, Bangladesh. \\ ${ }^{2}$ Department of Pharmacy, Atish Dipankar University of Science and Technology, Bangladesh.
}

\begin{abstract}
Ethyl acetate and chloroform partitionates of the ethanolic extracts of Aegle marmelos Linn. were screened for hypoglycemic activity in alloxan-induced diabetic rats $(200 \mathrm{mg} / \mathrm{kg}$, i.p.) and found to reduce blood glucose level significantly ( $p<0.05)$. The partitionates have also exhibited correction of altered biochemical parameters viz., cholesterol and triglycerides $(p<0.05)$. In this study, different fractions of $A$. marmelos to the alloxan-induced diabetic rats resulted in the significant elevation of liver glycogen content, which was decreased approximately 50\% in diabetic control. Liver enzymes, SGOT and SGPT levels were elevated in diabetic rats that were reduced after intraperitoneal administration of these fractions. These indicate that various fractions (ethyl acetate and chloroform) of the ethanolic extract of A. marmelos have favorable effects in bringing down the severity of diabetes together with hepatoprotectivity. The effect of these partitionates on the oral glucose tolerance test showed that glucose induced hyperglycemia can also be effectively reduced by them. The overall results showed that glucose level and plasma liver transaminases were increased and liver glycogen was decreased in diabetic rats, and that treatment with ethanolic extract partitionates of A. marmelos reversed the effects of diabetes on these biochemical parameters to near-normal levels.
\end{abstract}

Keywords-Fasting blood glucose (FBG), hepatoprotective, hypolipidemic, total cholesterol (TC), and triglyceride (TG).

\section{INTRODUCTION}

Diabetes mellitus is the world's largest endocrine disorder of multiple etiologies involving metabolic disorders of carbohydrate fat and protein. The aging populations, consumption of calorie-rich diets, obesity and sedentary lifestyles have led to a tremendous increase in the number of individuals with type 2 diabetes worldwide. According to World Health Organization projections, the prevalence of diabetes is likely to increase by $35 \%$ by the year $2025 .{ }^{[1]}$

At present the treatment of diabetes mainly involves a sustained reduction in hyperglycemia by the use of biguanides, thiazolidinediones, sulfonylureas, D-phenylalanine and $\alpha$-glucosidase inhibitors in addition to insulin. However, due to unwanted side effects the efficacies of these compounds are debatable and there is a demand for new compounds for the treatment of diabetes. ${ }^{[2],[3]}$ Hence plants have been suggested as a rich, as yet unexplored source of potentially useful antidiabetic drugs. Many traditional plants treatment for diabetes are used throughout the world. Plant drugs and herbal formulations are frequently considered to be less toxic and free from side effects than synthetic one. ${ }^{[4]}$

The attributed antihyperglycemic effect of these plants are due to their ability to restore the function of pancreatic tissues by causing an increase in insulin output or inhibit the intestinal absorption of glucose or to the facilitation of metabolites in insulin dependent processes. Hence, treatment with herbal drugs has an effect on protecting $\beta$-cells and smoothing out fluctuation in glucose levels. ${ }^{[5]}$ Since time immemorial, individuals with diabetes have been treated orally in folk medicine with a variety of plant extracts. Recently, there has been increasing interest in the use of medicinal plants. The World Health Organization has recommended especially in developing countries, the initiation of programs designed to use medicinal plants more effectively in the traditional healthcare system. ${ }^{[6]}$ 
Ethnobotanical information indicates that more than 800 plants have been used as traditional remedies for the treatment of diabetes. ${ }^{[7],[8]}$ The antihyperglycaemic activity of a large number of these plants has been evaluated and confirmed in different animal models. ${ }^{[9], ~[10], ~[11] ~}$

In the present study the antidiabetic properties of Aegle marmelos Linn. was assessed by evaluating the antihyperglycemic, hypolipidemic activities as well as their activities on the liver glycogen and liver enzymes (SGOT and SGPT) in alloxan-induced diabetic rats.

A. marmelos (locally named 'Bael'), a deciduous tree is widely distributed in this sub-continent including India, and Bangladesh. Its parts are principally used as a laxative, astringent, demulcent, stomachic, stimulant, antipyretic, antiscourbutic, haemostatic and aphrodisiac. Its leaves are advocated in the management of diabetes mellitus. [12], [13], [14], [15] So far limited reports are available on the antihyperglycemic activity of $A$. marmelos leaves with an unknown mode of action. Here we investigated the antidiabetic effects of ethyl acetate and chloroform partitionates of ethanolic extract of A. marmelos leaves in normal and alloxan induced diabetic rats.

\subsection{Plant Materials}

\section{MATERIALS AND METHODS}

Fresh leaves of A. marmelos was collected from medicinal plant garden, Department of Pharmacy, University of Rajshahi, Rajshahi and various parts of the locality. After thorough washing the leaves were dried completely under mild sun and ground in electric grinder into a coarse powder. The plants were authenticated by Mr. A.H.M. Mahbubur Rahman, Department of Botany, University of Rajshahi. A voucher specimen, collection \# 60, dated 8/25/2007 was kept in the Department of Botany, University of Rajshahi, Rajshahi.

\subsection{Preparation and fractionation of crude extracts}

The coarse powder was extracted by cold extraction technique in ethyl alcohol (95\%). The extracts of the plants obtained was the crude extracts. The crude extracts were dissolved in $100 \mathrm{ml}$ of water. To the solution almost equal volume of various organic solvents such as ethyl acetate, and chloroform were added sequentially. After proper shaking the layers were separated and dried under mild sun and the extracts so obtained was the final fractionated extract that was applied to the animal model for checking their antidiabetic activity.

\subsection{Drugs and Chemicals}

The active drug, metformin hydrochloride was the generous gift samples from Square Pharmaceuticals Ltd., Pabna Bangladesh. Total cholesterol (TC) and triglyceride (TG) wet reagent diagnostic kits were the products of Cresent diagnostic kits. Alloxan was purchased from Sisco Research Laboratories Pvt. Ltd. Mumbai, India. SGOT and SGPT wet reagent diagnostic kits were purchased from AMP Medizintechnik $\mathrm{GmbH}$; Austria.

\subsection{Selection of Animal}

The study was conducted on 50 Long Evans rats purchased from ICDDR, B, Dhaka. They were five to six weeks of age, weighing about 110-120 gm, which were housed in colony cages (six rats per cages) at an ambient temperature of $25-27^{\circ} \mathrm{C}$ with $12 \mathrm{hr}$ light and dark cycles having proper ventilation in the room .The rats were fed normal diets and water ad libitum. The animals were allowed to acclimatize to the laboratory environment for one week and then randomly divided into groups for experiments.

\subsection{Experimental induction of diabetes}

Animals were allowed to fast for $12 \mathrm{hr}$ and intraperitoneally administered freshly prepared alloxan (110 $\mathrm{mg} / \mathrm{kg}$ body weight) in saline water. Alloxan is a toxic reagent. It causes renal hypertrophy and severe abnormalities in renal transaminase and serum parameters: urea $\mathrm{N}$, creatinine, glutamic-oxaloacetic transaminase and glutamate-pyruvate transaminase activities in diabetic dogs. ${ }^{[16]}$ The alloxan treated animals were allowed to feed over night to overcome drug-induced hypoglycemic shock. After 48 - $72 \mathrm{hr}$ (to allow for the development and aggravation of diabetes), rats with moderate diabetes having persistent glycosuria and hyperglycemia were considered diabetic for further experimentation. ${ }^{[17]}$

\subsection{Experimental design}

In the experiment, a total of 50 rats (20 diabetic surviving rats, 30 normal rats) were used. Group II to Group V was prepared for testing antihyperglycemic effects after chemical diabetes. Group I receives only vehicle (DMSO). Group II was selected for diabetic control, which does not receive either metformin, or plant extracts. Group III stands for metformin control group in which metformin was administered intraperitoneally at a dose of $150 \mathrm{mg} / \mathrm{kg}$ body weight. Group IV and V received ethyl acetate and chloroform partitionates of $A$. marmelos leaf extracts. The blood samples were analyzed for blood glucose content at $0,2,6,16$, and 24 hours, respectively after treatment. For Oral glucose tolerance test, (OGTT) animals were grouped in five groups. Group VI to Group X was prepared for testing hypoglycemic effects after glucose-induced hyperglycemia in rats. Group VI receives only vehicle (DMSO). Group VII was selected for positive control, which does not receive either metformin, or plant extracts. Group VIII stands for metformin control. Group IX and X received ethyl acetate and chloroform partitionates of A. marmelos leaf extracts. After overnight fasting, a baseline blood glucose level was estimated ( 0 minutes). Without delay, a glucose solution ( $\mathrm{gm} / \mathrm{kg}$ body weight) was 
administered by gavage. At the same time standard drug and plant extracts were administered intraperitoneally to the respective animal groups. Five more blood samples were taken at 30, 60, 90, 150, and 270 minutes after glucose administration and blood glucose level was estimated in all the experiments by using glucometer (Bioland-423, Germany).

\subsection{Collection of serum and determination of serum Total Cholesterol (TC), serum Triglycerides (TG), SGOT and SGPT}

After completing blood glucose level estimation, rats were sacrificed and about 3-5 $\mathrm{ml}$ of blood was collected directly from heart by syringes, centrifuged at $4000 \mathrm{rpm}$ for 10 minutes and the serum was preserved to examine, TC, TG, SGOT and SGPT concentrations by UV spectrophotometric method (Shimidzu UV-1200, Tokyo, Japan), using wet reagent diagnostic kits according to manufacturer's protocol. At the same time liver tissues were also collected for the estimation of glycogen content in liver.

\subsection{Estimation of glycogen content in liver}

Glycogen content in liver was measured according to spectrophotometric determination of glycogen with o-toluidine reagent. It utilizes the o-toluidine glucose coupling reactions for the estimation of glycogen after trichloroacetic acid extraction, precipitation by alcohol and hydrolysis.

\subsection{Phytochemical screening methods}

Phytochemical tests have been performed according to the literature by Nayak and Pereira. ${ }^{[18]}$

\subsubsection{Test for saponins}

$300 \mathrm{mg}$ of extract was boiled with $5 \mathrm{ml}$ water for two minutes. The mixture was cooled and mixed vigorously and left for three minutes. The formation of frothing indicated the presence of saponins.

\subsubsection{Test for tannins}

To an aliquot of the extract sodium chloride was added to make to $2 \%$ strength. Then it is filtered and mixed with $1 \%$ gelatin solution. Precipitation indicated the presence of tannins.

\subsubsection{Test for triterpenes}

$300 \mathrm{mg}$ of extract was mixed with $5 \mathrm{ml}$ chloroform and warmed for 30 minutes. The chloroform solution was then treated with a small volume of concentrated sulphuric acid and mixed properly. The appearance of red color indicated the presence of triterpenes.

\subsubsection{Test for alkaloids}

$300 \mathrm{mg}$ of extract was digested with $2 \mathrm{M} \mathrm{HCl}$. Acidic filtrate was mixed with amyl alcohol at room temperature, and examined the alcoholic layer for the pink color that indicated the presence of alkaloids.

\subsubsection{Test for flavonoids}

The presence of flavonoids was determined using $1 \%$ aluminium chloride solution in methanol, concentrated $\mathrm{HCl}$, magnesium turnins, and potassium hydroxide solution.

\subsection{Statistical Analysis}

Statistical comparisons were performed by one-way analysis of variance (ANOVA), followed by Scheffe's post-hoc test or students paired or unpaired $t$-test where appropriate. Results are considered to be significant when $\mathrm{p}$ values were less than $0.05(p<0.05)$. Statistical calculations and the graphs are prepared using GraphPad Prism version 4.00 for Windows (GraphPad Software, San Diego, CA, USA, www.graphpad.com). The results are expressed as mean \pm SEM.

\section{RESULTS}

The effect of the different fractions of A. marmelos on the fasting blood glucose (FBG) level, serum total cholesterol (TC), serum triglyceride (TG), SGOT, SGPT levels and glycogen content in liver were investigated in the control and alloxan-induced diabetic rats using metformin $\mathrm{HCl}$ as standard antidiabetic agents.

\subsection{Effect of different fractions of A. marmelos on FBG level in diabetic rats}

The mean blood glucose concentration of control and different fractions of $A$. marmelos-treated animals were estimated on the 2, 6, 16, and 24 hours, respectively as shown in Fig 1.Their baseline glucose concentrations were also measured ( 0 hour). Ethyl acetate fraction of $A$. marmelos reduced blood glucose level to $77.78 \pm 6.9 \%, 51.59 \pm 1.9 \%, 23.01 \pm 4.5 \%$, and $32.54 \pm 2.5 \%$ at $2,6,16$ and 24 hours, respectively. Maximum reduction of blood glucose level of $76.99 \%$ was observed on 16 hour of the experiment. Chloroform fraction of $A$. marmelos showed reduction of blood glucose level to $85.92 \pm 5.45 \%, 72.39 \pm 4.85 \%, 25.91 \pm$ $5.94 \%$ and $40.56 \pm 1.3 \%$ at 2, 6, 16 and 24 hours, respectively. Maximum reduction of blood glucose level of $74.09 \%$ was also observed on 16 hour during the 24-hour experimental period. In case of alloxan induced diabetic rats metformin reduced blood glucose level to $68.50 \pm 1.10 \%, 38.43 \pm 1.04 \%, 20.10 \pm 3.0 \%, 24.91 \pm$ $2.5 \%$ at 2, 6, 16 and 24 hours, respectively. So metformin caused maximum reduction of blood glucose level of $79.90 \%$ on 16 hour of the experiment.

\subsection{Effect of different fractions of A. marmelos on TC and TG levels in diabetic rats}

The mean serum TC and TG levels of control and treated animals after 24 hours are shown in Fig. 2 
and Fig. 3, respectively. Hypolipidemia was observed in animals treated with the different fractions. In the case of the effects of standard metformin $\mathrm{HCl}$ and different fractions of A. marmelos on TC level in diabetic rats, the metformin, ethyl acetate and chloroform fractions reduced the TC level to $46.55 \pm 3.87 \%, 22.52 \pm$ $2.60 \%$ and $39.38 \pm 5.62 \%$, respectively. Maximum reduction of serum TC level of $77.48 \%$ was found for ethyl acetate fraction. During the effects of metformin and different fractions of $A$. marmelos on serum TG level in diabetic rats, the metformin, ethyl acetate and chloroform fractions reduced the serum TG level to $38.98 \pm 4.53 \%, 53.09 \pm 2.9 \%$, and $38.98 \pm 3.82 \%$, respectively. Maximum reduction of $61.02 \%$ was observed for ethyl acetate fraction of $A$. marmelos.

\subsection{Effect of experimental plant fractions on the level of glycogen content in diabetic rats}

In this study, it is found that the level of glycogen in liver was reduced to $49.34 \pm 4.25 \%$ in diabetic rats as compared to the normal control group. Treatment of diabetic rats with metformin standard, ethyl acetate and chloroform fractions of $A$. marmelos the level of glycogen content was improved to $86.31 \pm 2.49 \%, 80.19 \pm$ $1.98 \%$ and $97.77 \pm 3.43 \%$, respectively as shown in the Fig. 4. In this case chloroform fraction had more significant activity in glycogen synthesis.

3.4 Effect of different fractions of A. marmelos on liver enzymes (SGOT, SGPT) in diabetic rats

In diabetic rats, SGOT and SGPT levels were raised to $60.86 \pm 2.74 \%$ and $19.85 \pm 4.67 \%$, respectively in comparison to normal rats. Intraperitoneal administration of different plant fractions decreased the liver enzymes in diabetic animals to reestablish the normal condition as shown in the Table 1.

\subsection{Effect of different fractions of A. marmelos on FBG level in the glucose-induced hyperglycemic rats}

Ethyl acetate fraction of $A$. marmelos showed reduction of blood glucose level to $83.63 \%, 82.53 \%$, and $83.10 \%$ and chloroform fraction of $A$. marmelos reduced blood glucose level to $73.63 \%, 77.24 \%$, and $89.86 \%$ at $30 \mathrm{~min}, 60 \mathrm{~min}$, and $90 \mathrm{~min}$, respectively in glucose induced hyperglycemic rats as shown in the Fig. 5. So it was found that ethyl acetate fraction showed maximum reduction of $17.47 \%$ at 90 min of the experiment and chloroform fraction, $26.37 \%$ at 30 min during the experimental period.

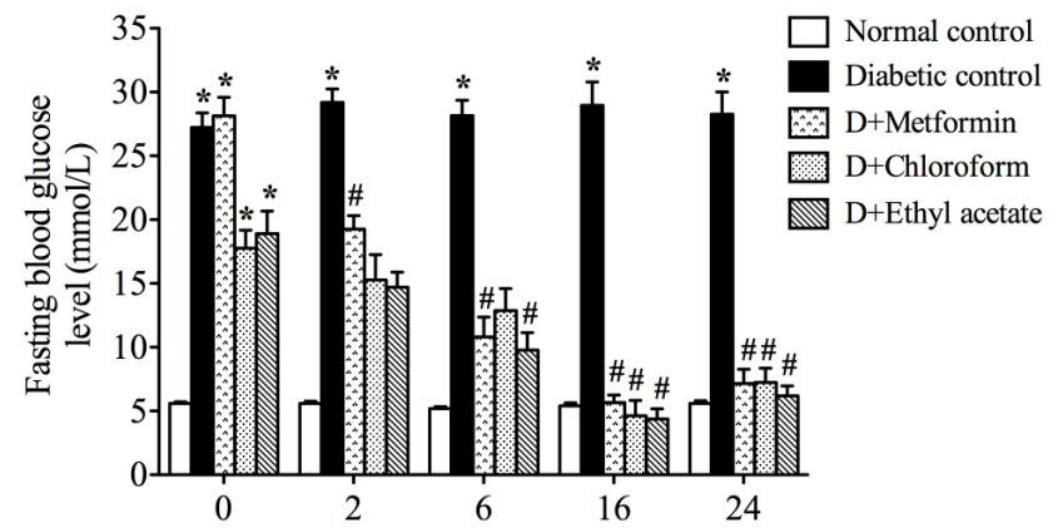

Fig. 1 Effect of different fractions of A. marmelos on the FBG level on diabetic rats compared to normal rats. * indicates significant changes (increase) of blood glucose level compared with normal control group. \# indicates significant changes (decrease) of FBG level in diabetic rats after treatment compared with zero hour treatment group. The results are expressed as means \pm SEM.

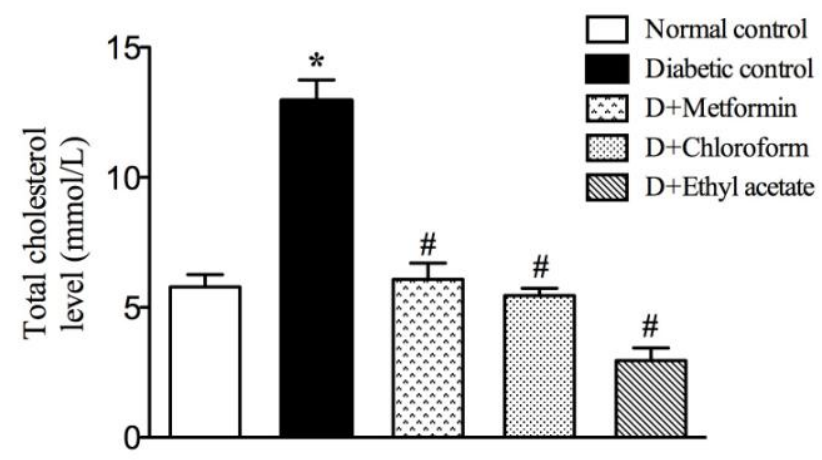

Fig. 2 Effect of different fractions of A. marmelos on the serum TC level in diabetic rats. * indicates significant changes (increase) of TC level compared with normal control group. \# indicates significant changes (decrease) in diabetic rats after treatment. 


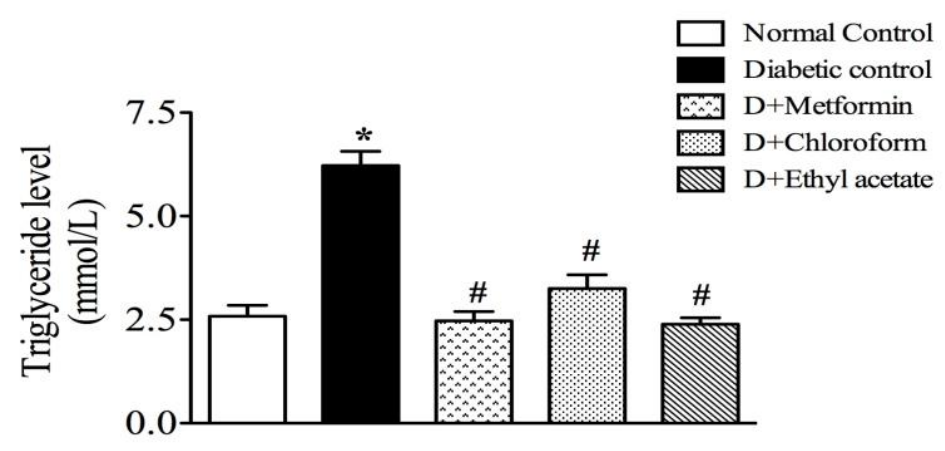

Fig. 3 Effect of different fractions of A. marmelos on the serum TG level in diabetic rats. * indicates significant changes (increase) of TG level compared with normal control group. \# indicates significant changes (decrease) in diabetic rats after treatment.

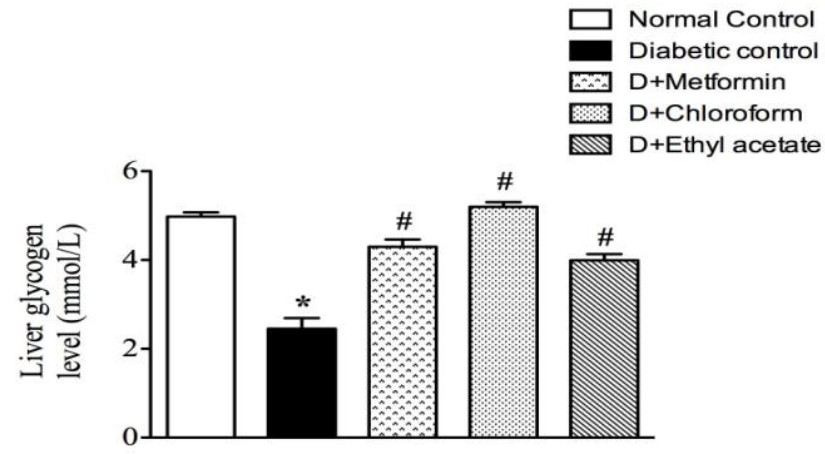

Fig. 4 Effect of different fractions of A. marmelos on the liver glycogen content in diabetic rats after treatment. * indicates significant decrease of liver glycogen content after diabetes induction compared to normal control. Glycogen level was again reestablished to nearly normal after treatment with plant fractions.

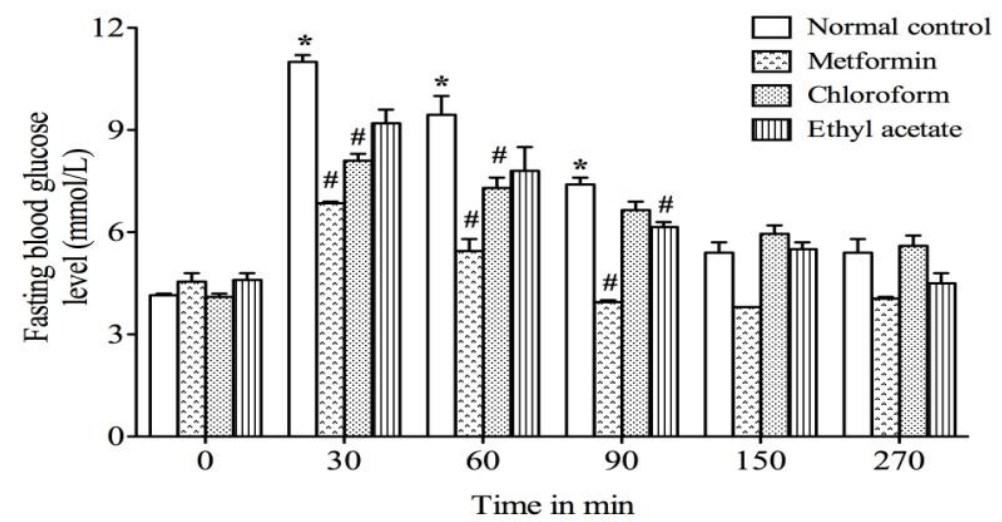

Fig. 5 Effect of different fractions of A. marmelos on the glucose-induced hyperglycemia in normal rats. Glucose induced hyperglycemia was significantly decreased after treatment. * indicates glucose induced hyperglycemia and \# indicates significant reduction after treatment.

Table 1. The phytochemical constituents of the experimental plant fractions obtained by phytochemical screening tests (+ indicates presence and - indicates absences of the constituents.)

\begin{tabular}{llllll}
\hline Partitionates & Saponin & Tannins & Triterpines & Alkaloids & Flavonoids \\
& & & & & + \\
\hline Chloroform & - & + & - & + & + \\
\hline Ethyl acetate & - & + & + & + & + \\
\hline
\end{tabular}


Table 2. Effect of different fractions of A. marmelos ethanolic extract on SGOT and SGPT level. * indicates significant difference $(p<0.05)$ from normal control group. \# indicates significant difference $(p<0.05)$ from the diabetic control. Data are expressed as means \pm SEM.

\begin{tabular}{lcc}
\hline Group & \multicolumn{1}{c}{$\begin{array}{c}\text { SGOT } \\
(\text { Unit/ml) }\end{array}$} & $\begin{array}{c}\text { SGPT } \\
(\text { Unit/ml) }\end{array}$ \\
\hline Normal Control & $18.0 \pm 1.9$ & $21.4 \pm 1.73$ \\
Diabetic Control & $46.0 \pm 2.35^{*}$ & $26.7 \pm 4.32$ \\
D+Metformin & $25.2 \pm 3.17 \#$ & $11.5 \pm 2.31 \#$ \\
D+Chloroform & $21.6 \pm 1.79 \#$ & $21.6 \pm 0.67 \#$ \\
\hline D+Ethyl acetate & $13.0 \pm 0.97 \#$ & $13.0 \pm 1.45 \#$ \\
\hline
\end{tabular}

\section{DISCUSSION}

Diabetes mellitus is possibly the world's largest growing metabolic disorder, and as the knowledge on the heterogeneity of this disorder is advanced, the need for more appropriate therapy increases. ${ }^{[19]}$ Traditional plant medicines are used throughout the world for a range of diabetic presentations. The study of such medicines might offer a natural key to unlock a diabetologist's pharmacy for the future.

In the light of the literature on A. marmelos, we made an attempt for the first time to study the effect of A. marmelos ethanolic extract partitionates in normoglycemic and hyperglycemic rats. The significant antidiabetic activity of ethyl acetate and chloroform fraction of A. marmelos as shown in Fig. 1 may be due to the presence of hypoglycemic alkaloids, saponins, triterpines, and flavonoids. It could be conceived that the plant extracts may also contain some biomolecules that may sensitize the insulin receptor to insulin or stimulates the $\beta$-cells of islets of langerhans to release insulin which may finally lead to improvement of carbohydrate metabolizing enzymes towards the re-establishment of normal blood glucose level.

Hypercholesterolemia and hypertriglyceridemia have been reported to occur in diabetic rats. [20], [21],

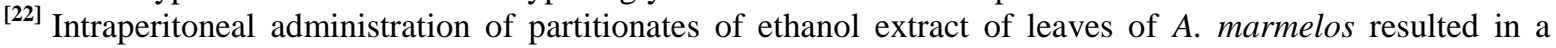
significant reduction of serum lipid levels in rats with hyperlipidemia viz. total cholesterol and triglyceride (Fig. 2 and 3). Flavonoids are known for their diverse biological activities including hypolipidemic activity resulting from their antioxidant activity. ${ }^{[23]}$ A. marmelos partitionates showed the presence of flavonoids and related phenolic compounds (Table 1). Such dual property has also been reported in methanol extract of Prunus davidiana (Rosaceae) and its flavonoid constituent, Prunin. ${ }^{\text {[24] }}$

Induction of diabetes with alloxan was associated with decrease in hepatic glycogen, which could be attributed to the decrease in the availability of the active form of enzyme glycogen synthetase probably because of low levels of insulin. ${ }^{[25], ~[26] ~ I n ~ t h e ~ p r e s e n t ~ s t u d y, ~ A . ~ m a r m e l o s ~ r e s t o r e d ~ t h e ~ d e p r e s s e d ~ h e p a t i c ~}$ glycogen levels possibly by increasing the level of insulin. Our results showed that supplementation of diabetic rats with plant fractions resulted in significant elevation in hepatic glycogen content. Decreased in the activities of the enzymes involved in glucose homeostasis in liver and kidney such as hexokinase has been reported in diabetic animals resulting in depletion of liver and muscle glycogen content. ${ }^{[27]}$ Treatment with plant extracts might increase the level of enzyme to the control level indicating an over-all increase in glucose influx. The exact mechanism of action needs further investigation.

There was a significant rise in serum GOT and GPT levels in diabetic rats, which could relate to excessive accumulation of amino acids (glutamate and alanine) in the serum of diabetic animals as a result of amino acids mobilization from protein stores. ${ }^{[28]}$ The higher levels of GOT and GPT, may give rise to a high concentration of glucose. In other words, the gluconeogenic action of GOT and GPT plays the role of providing new supplies of glucose from other sources such as amino acids. Following intraperitoneal administration of different plant fractions, SGOT and SGPT levels were significantly reduced (Table 2).

Oral glucose tolerance test (OGTT) measures the body's ability to use glucose, the body's main source of energy. ${ }^{[29]}$ It can be used to diagnose prediabetes and diabetes. In our study, it is found that various fractions have also hypoglycemic effect in glucose induced hyperglycemic rats. This may be due to the presence of hypoglycemic alkaloids, flavonoids, triterpines or saponin glycosides that also requires further investigation.

\section{CONCLUSION}

This study is unique in that plant fractions cause rapid induction of hypoglycemia and hypolipidemia as well as possesses hepatoprotective effect in diabetic rats. In the light of our pharmacological studies $A$. marmelos leaf extracts can be useful, at least as an adjunct, in the therapy of diabetes, a condition in which hyperglycemia and hyperlipidemia coexist quite often. We need further study to determine the mechanism of action and to isolate the active principles responsible for antidiabetic activity. 


\section{ACKNOWLEDGEMENTS}

The present work was supported by the National Science and Information and Communication Technology (NSICT), Dhaka, Bangladesh for financial assistance to the first author and the authors would like to extend their gratitude to the Director, Animal Research Centre (ARC), ICDDR, B for providing necessary facilities.

\section{REFERENCES}

[1] J.P. Boyle, A.A. Honeycutt, K.M. Narayan, T.J. Hoerger, L.S. Geiss, H Chen, and T.J. Thompson, Projection of diabetes burden through 2050: Impact of changing demography and disease prevalence in the U.S, Diabetes Care, 24(11), 2001, 1936-1940.

[2] U.K Prospective Diabetes Study Group, Overview of six years therapy of type 2 diabetes: a progressive disease, Diabetes, 44, 1995, 1249-1258.

[3] D.E. Moller, New drug targets for type 2 diabetes and the metabolic syndrome, Nature, 414, 2001, 821-827.

[4] C.J. Bailey, and C. Day, Traditional treatments for diabetes, Diabetes Care, 12, 1989, 553-564.

[5] W. Jia, W.Y. Gao, and P.G. Xiao, Antidiabetic drugs of plant origin used in China, Composition, pharmacology, and hypoglycemic mechanisms. Zhonggue Zhong Yao Za Zhi, 28, 2003, 108-113.

[6] World Health Organization Resolution, Geneva, Switzerland, Drug Policies and Management, Medicinal Plants, 1978, 31-33.

[7] S.S. Ajgaonkar, Herbal drugs in the treatment of diabetes. International Diabetes Federation Bulletin, 24, 1979, $10-17$.

[8] F.J. Alarcon-Aguilara, R. Roman-Ramos, S. Perez-Gutierrez, A. Aguilar-Contreras, C.C Contreras-Weber \& J.L. Flores-Saenz, Study of the anti-hyperglycemic effect of plants used as antidiabetics, J. Ethnopharmacol, 61, 1998, $101-110$.

[9] I.B. Farjou, M. Al-Anna \& S.Y. Guirgues, Lowering of blood glucose in diabetic rabbits by Artemisia extract, $J$. Faculty of Medicine, Baghdad, 92, 1987, 137-141.

[10] S.K Swanston-Flatt, C. Day, P.R. Flatt \& C.J. Bailey, Evaluation of the antihyperglycaemic properties of traditional plant treatment for diabetes, In: Frontiers in Diabetes Research: Lessons from Animal Diabetes, 1991, 286-293.

[11] H. Jouard, M. Eddouks, M.A. Lacaille-Dubois \& B. Lyoussi, Hypoglycemic effect of the water extracts of Spergularia purpurea in normal and streptozotocin-induced diabetes rats, J. Ethnopharmacol, 71, 2000, 169-177.

[12] M.M. Alam, M.B. Siddiqui, and W. Husain, Treatment of diabetes through herbal drugs in rural India, Fitoterapia, 61, 1990, 240-242.

[13] V. Prakash, Diabetes management-some useful herbs, Jeevaniya Shishir, 4, 1992, 10-18.

[14] V.V Rao, Studies on hypoglycemic activity of some indigenous drugs in normal and alloxan induced diabetic rabbits, M.V. Sc. diss., IVRI Deemed University Izatnagar, India, 1992.

[15] P.T.C Ponnachan, C.S. Paulose, and K.P. Panikar, Effect of leaf extract of Aegle marmelos in diabetic rats, Indian Journal of experimental Biology, 31, 1993, 345-347.

[16] R. Cutfield, D.S. Albu, I.F. Chan, F. Moatame, G.L. Peric, and D.E. Wilson, Horm. Metab. Res., 16, 1984, 549550 .

[17] S. Lenzen, The mechanisms of Alloxan- and streptozotocin- induced diabetes, Diabetologia, 51, 2008, 216-226.

[18] B. S. Nayak, and L. M. Pinto Pereira, Catharanthus roseus flower extract has wound-healing activity in Sprague Dawley rats, BMC Complement Altern. Med., 6, 2006, 41.

[19] C.J. Baily, P.R. Flatt, Antidiabetic drugs, new developments, Indian Biotechnology, 6, 1986, 139-142.

[20] A. Riyad, Abdul-Ghani, S. Abdul-Salam, S.M. Suleiman, Effect of Fenugreek and Lupine Seeds on the Development of Experimental Diabetes in Rats, Planta Med, 54, 1988, 286-290.

[21] S.P. Tarfa, P.K. Joseph, K.T. Augusti, Curr Sci., 57, 1988, 32.

[22] S.R. Sharma, S.K. Dwivewdi, D. Swarup, Indian J Exp Biol, 34, 1996, 372.

[23] I.B. Afanas'ev, E.A. Ostrachovitch, N.E. Abramova, L.G. Korkina, Different antioxidant activities of biflavonoid rutin in normal and iron overloading rats, Biochem. Pharmacol., 80, 1995, 627-635.

[24] J.S. Choi, O.H. Yokozawa, Antihyperlipidemic Effect of Flavonoids from Prunus davidiana, J Nat Prod., 54, 1991, 218-224.

[25] A.H. Gold, The effect of diabetes and insulin on liver glycogen synthetase activation. J. Biol. Chem., 245, 1970, 903-905.

[26] R.K. Goel, M.P. Mahajan, and S.K. Kulkarni, Evaluation of antihyperglycemic activity of some novel monocyclic beta lactams, J. Pharmacy and Pharmaceutical Sciences, 7, 2004, 80-83.

[27] J.K. Grover, V.Vats and S.S. Rathi, Antihyperglycemic effect of Eugenia jambolana and Tinospora cardifolia in experimental diabetes and their effects on key metabolic enzymes involved in carbohydrate metabolism, $J$. Ethnopharmacol., 73(3), 2000, 461-470.

[28] V. Colev, M. Badescu, and I. Paduraru, The zinc metabolic disorder relation in experimental diabetes mellitus, Rom J. Inter. Med., 32, 1994, 71- 75.

[29] V. Du Vigneaud, W.G. Karr, Carbohydrate utilization, rate of disappearance of D-glucose from the blood, J. Biol. Chem., 66, 1925, 281-300. 